\title{
Erratum: Critical properties of a two-dimensional Ising magnet with quasiperiodic interactions [Phys. Rev. E 93, 042111 (2016)]
}

\author{
G. A. Alves, M. S. Vasconcelos, and T. F. A. Alves \\ (Received 3 June 2016; published 13 July 2016)
}

DOI: 10.1103/PhysRevE.94.019904

In our paper, we have determined all parameters [EdwardsAnderson (EA) order parameter $\left\langle q_{E A}\right\rangle$, the susceptibility $\chi$, the specific heat $c$, and Binder cumulant $\left.g_{L}\right]$ by using the standard Monte Carlo method together with the Metropolis algorithm. Also, we have concluded that, at low temperature, we have a spin glass phase. In this case, by using the standard Monte Carlo algorithm (MCA), it is expected to let the system fall, at low temperatures, in a minimum of energy. However, our system, at low temperatures, could have many local minima of energies [1]. Therefore, applying the MCA could let the system fall in a local minimum of energy that corresponds to a metastable state. In order to avoid this we use the replica exchange Monte Carlo technique [1-4], which is more suited to approach spin glasses instead of the MCA, and we conclude that the numerical values presented in the original paper are wrong. They could represent some metastable phase of the system.

Therefore, our numerical results should be corrected in the original article. Then, by using the replica exchange Monte Carlo technique we have obtained a correct estimate of the critical temperature, that is around $T_{c} \approx 1.268$ (less than the previous wrong value by $44 \%$ ) as showing in Fig. 3 (which replaces Fig. 3 of our original article). The correspondent order parameter is now shown in Fig. 4 (which replaces Fig. 4 of our original article).

The susceptibility and the specific heat are shown in Figs. 7 and 10 (which replace Figs. 7 and 10 of our original article).

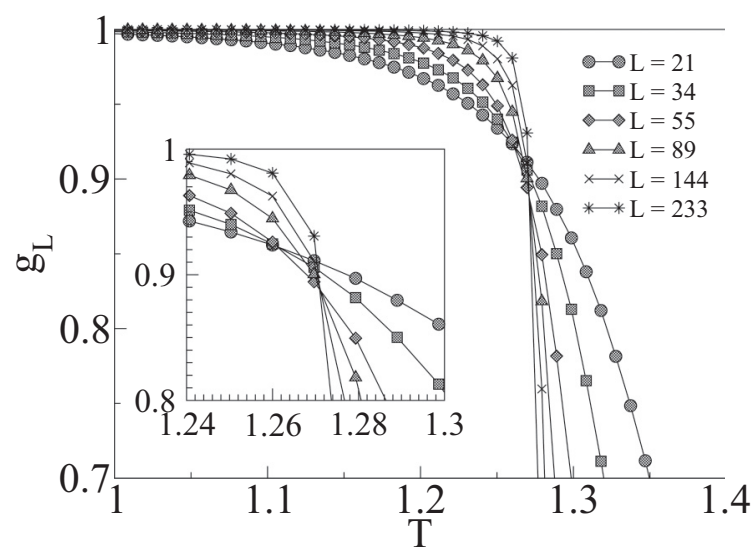

FIG. 3. Binder cumulant $g_{L}$ vs temperature $T$ for different lattice sizes $L$. The values of $L$ obey the Fibonacci sequence. We estimated the critical temperature $T_{c} \approx 1.268$ by averaging the numerical values of the temperatures where the curves intersect each other. We have, for this model, a phase transition from a paramagnetic phase to a spin-glass phase by decreasing the temperature.

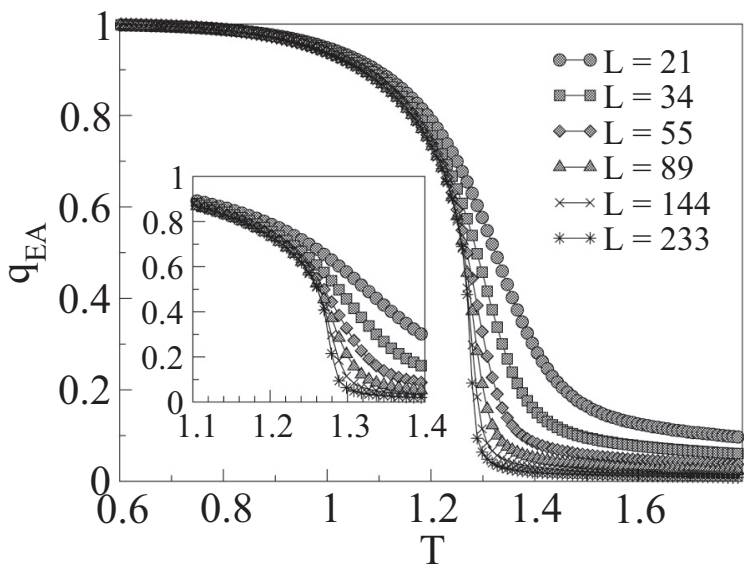

FIG. 4. The EA order parameter $q_{E A}$ as a function of temperature $T$ for different lattice sizes $L$. The values of $L$ obey the Fibonacci sequence. The curves suggest a second order phase transition.

We would like to emphasize from those corrected figures that the corrected numerical results do not affect the conclusions of our original paper. Only the critical temperature and the critical exponents must be corrected.

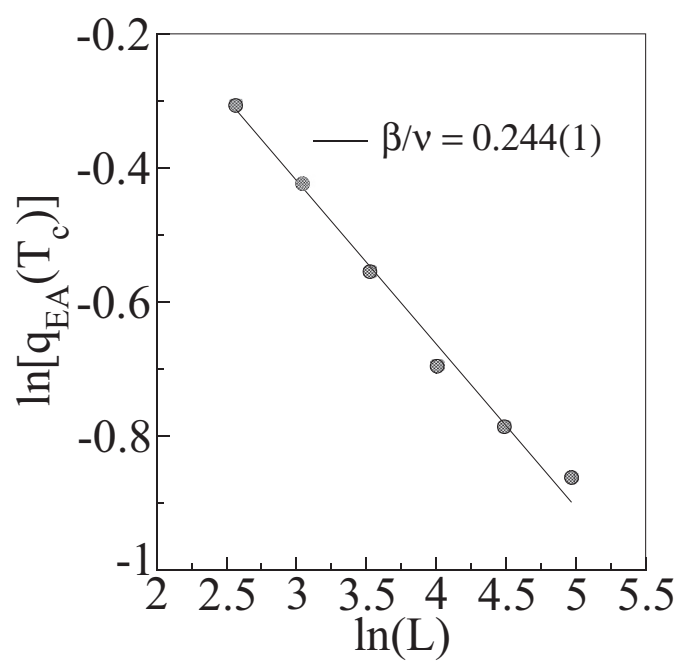

FIG. 5. Critical behavior of $q_{E A}$ at $T=T_{c}$ as a function of lattice size $L$ obtained from Eq. (6) of our original article. Alongside the $q_{E A}$ points we show the error bars on the same scale. The curve slope gives the exponent ratio $\beta / v=0.24(1)$. The exponent ratio differs from the pure model and this change of the universality class is induced by the quasiperiodic ordering. 


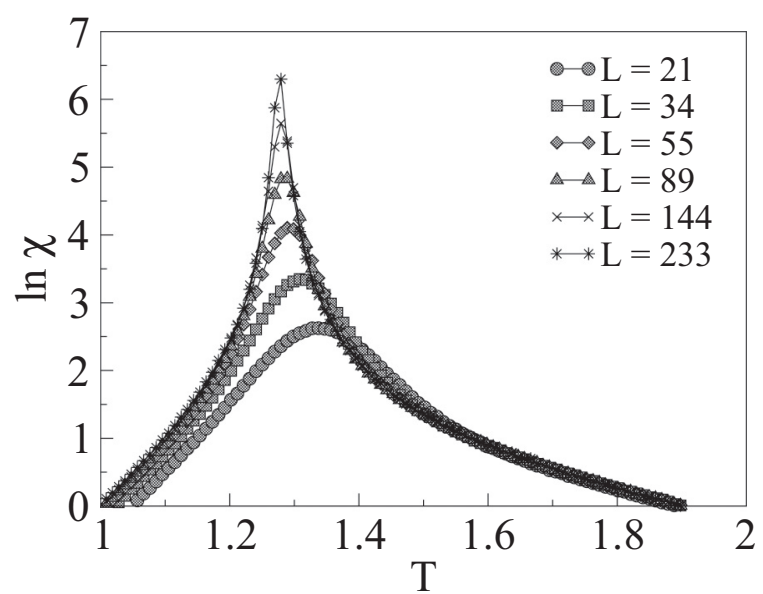

FIG. 7. The susceptibility $\chi$ as a function of temperature $T$ for different lattice sizes $L$. The values of $L$ obey the Fibonacci sequence. The susceptibility diverges at $T_{c}$ in the large lattice size limit suggesting a second order phase transition.

The news critical exponents, calculated by using the replica exchange Monte Carlo technique, are $\beta / \nu=0.24(1)$ (that is less than the previous wrong value by $40 \%), \gamma / v=1.50(1)$ (it is greater than the previous wrong value by $17 \%$ ), and $1 / v=0.89$ (3) (it exceeds the previous wrong value by $6 \%$ ). Those values are calculated from a linear fit shown in Figs. 5, 8, and 9 (which replace Figs. 5, 8, and 9 of our original article, respectively).

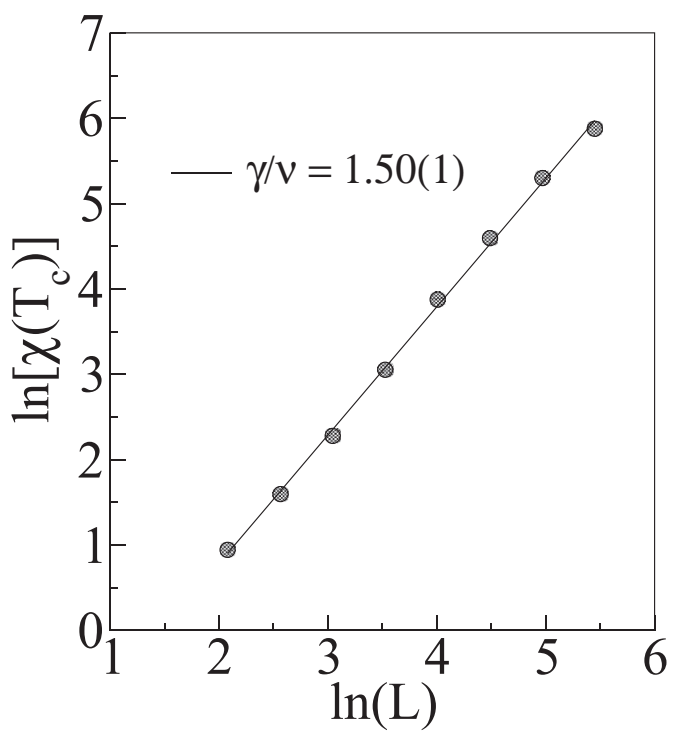

FIG. 8. Critical behavior of $\chi$ at $T=T_{c}$ as a function of lattice size $L$ obtained from Eq. (7) of our original article. Alongside the $\chi$ points we show the error bars on the same scale. The curve slope gives the exponent ratio $\gamma / v=1.50(1)$, differing from the pure Ising $2 \mathrm{D}$ case.

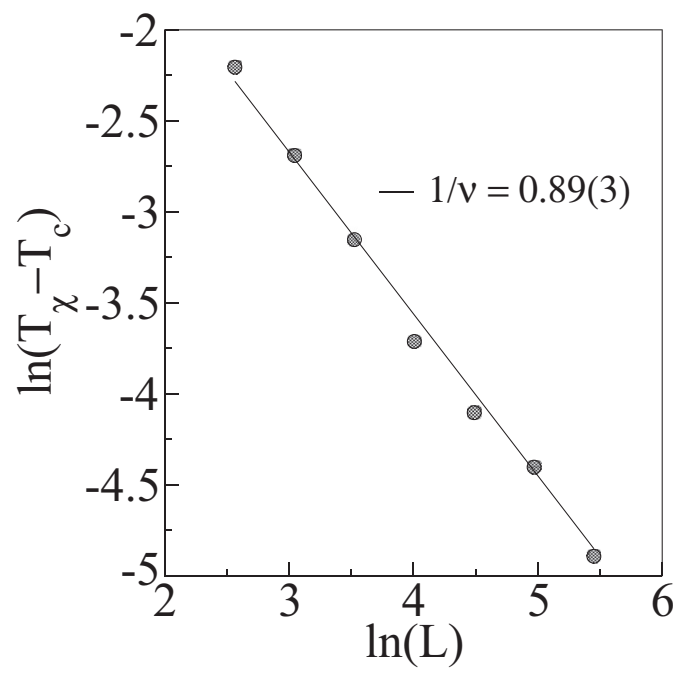

FIG. 9. Critical behavior of susceptibility maximum temperatures $T_{\chi}$ as a function of lattice size $L$ obtained from Eq. (10) of our original article. The curve slope gives the exponent $1 / v=0.89(3)$, differing from the pure Ising $2 \mathrm{D}$ case.

Finally, we have provided the data collapses of $q_{E A}$ order parameter, susceptibility, and specific heat in Figs. 11 and 12 (these figures replace Figs. 11 and 12 of our original article, respectively). From those results we obtain $\alpha / v \approx-0.24$ (less than the previous wrong value by $40 \%$ ) by a data collapse for the specific heat, shown in Fig. 11. We still found a spin glass phase at finite temperatures with different exponents from the two-dimensional (2D) Ising universality

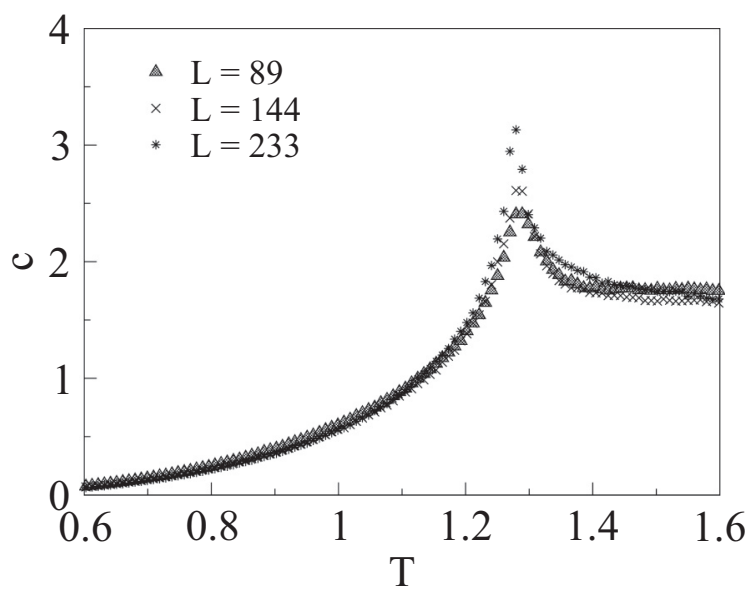

FIG. 10. Specific heat $c$ as a function of temperature $T$ for different lattice sizes $L$. The values of $L$ obey the Fibonacci sequence. When increasing the lattice size, we observe a crescent maximum, suggesting a logarithm divergence or a negative exponent divergence at the critical temperature $T_{c} \approx 1.268$. 


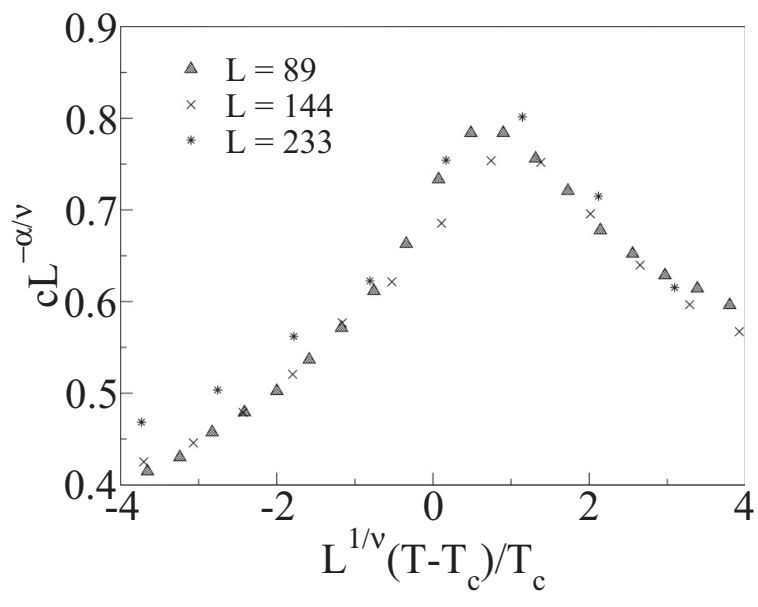

FIG. 11. Data collapse of specific heat $c$ for different lattice sizes $L$. The best data collapse gives us the estimate for $\alpha / v \approx-0.24$.

class, but with a corrected estimate of the critical temperature around $T_{c} \approx 1.268$ and the exponent $\beta=0.27$ (1) (less than the previous wrong value by $43 \%$ ).
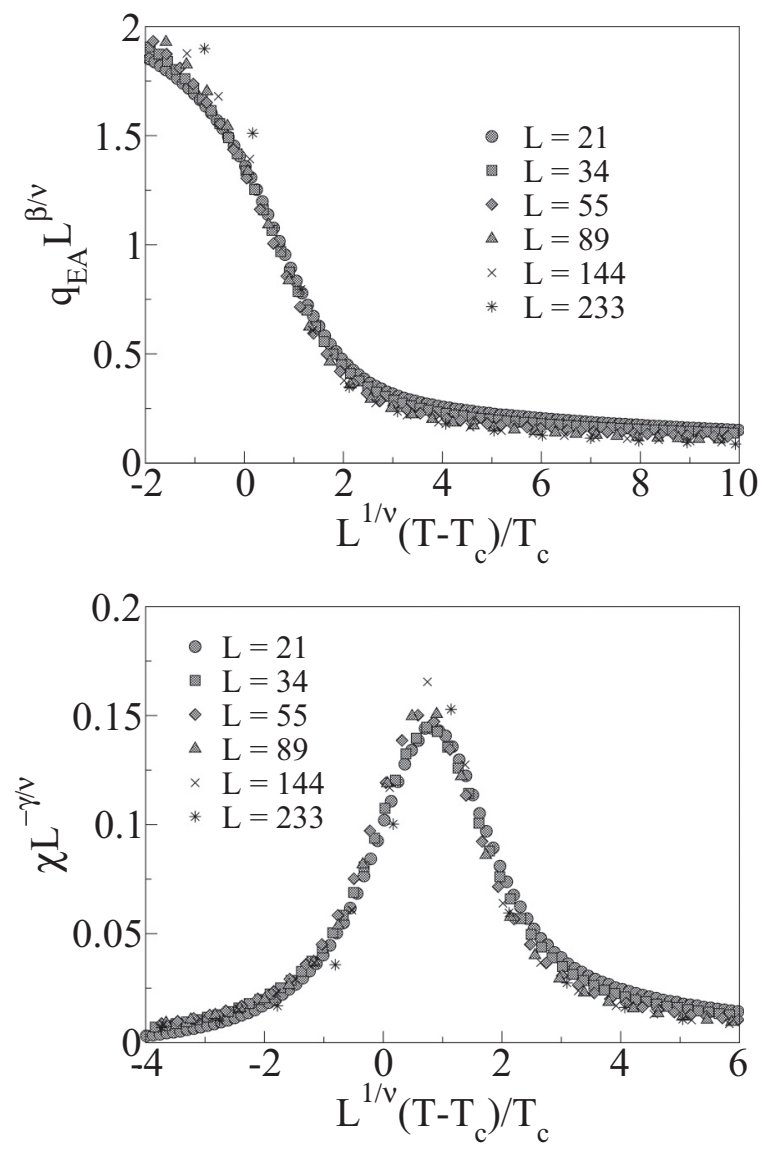

FIG. 12. Data collapse of EA order parameter $q_{E A}$ and susceptibility $\chi$. The thermodynamic parameters as functions of lattice sizes collapse for $\beta / v=0.24(1), \gamma / v=1.50(1)$, and $1 / v=0.89$ (3) next to the critical temperature according to the scale forms given in Eqs. (6) and (7) of our original article differing from the pure Ising 2D case.
[1] K. Hukushima and K. Nemoto, J. Phys. Soc. Jpn. 65, 1604 (1996).

[2] D. J. Earl and M. W. Deem, Phys. Chem. Chem. Phys. 7, 3910 (2005).
[3] R. H. Swendsen and J.-S. Wang, Phys. Rev. Lett. 57, 2607 (1986).

[4] C. J. Geyer, in Computing Science and Statistics Proceedings of the 23rd Symposium on the Interface (American Statistical Association, Virginia, 1991), pp. 156-163. 